\title{
A VARIATIONAL MODEL FOR URBAN PLANNING WITH TRAFFIC CONGESTION
}

\author{
${\text { Guillaume } \text { CARlier }^{1} \text { and Filippo Santambrogio }}^{2}$
}

\begin{abstract}
We propose a variational model to describe the optimal distributions of residents and services in an urban area. The functional to be minimized involves an overall transportation cost taking into account congestion effects and two aditional terms which penalize concentration of residents and dispersion of services. We study regularity properties of the minimizers and treat in details some examples.
\end{abstract}

Mathematics Subject Classification. 49K20, 49K30.

Received July 15, 2004.

\section{INTRODUCTION}

Mathematical models for optimal urban planning may raise challenging questions both from the theoretical and applied point of view. Such models may be traced back to the work of Beckmann [2] who, in the early 50's, introduced the so-called continuous transportation model in urban economics, leading to a minimal flow like problem. Roughly speaking, the minimal flow problem consists in finding a vector-field with prescribed divergence with minimal $L^{1}$ norm. For such problems, one of course has to properly define generalized (i.e. vector-valued measures) solutions, and this can be achieved by using the recent developments in the $L^{1}$ optimal transportation theory in particular the notion of transport density (see de Pascale and Pratelli [7]). More generally, thanks to recent advances in optimal transportation theory, new variational models in optimal urban planning have emerged: for instance Buttazzo and Stepanov [6] studied the design of an optimal transportation network in a city with given densities of populations and workplaces. In [5], Buttazzo and the second author proposed a model to describe the optimal distributions of residents and services in a prescribed area by minimizing a cost functional taking into account the transportation costs (according to a Monge-Kantorovich type criterion) and two additional terms which penalize concentration of residents and dispersion of services (see also $[10])$.

As in [5], given a urban area $\Omega$ (a subset of $\mathbb{R}^{2}$ in the applications), we look for the distribution of residents (or consumption), denoted by $\mu$, and the distribution of services (or production), denoted by $\nu$, so as to minimize a cost involving three terms: an overall transportation term for moving customers to services, a term penalizing dispersion of services and a term penalizing concentration of residents. We mainly depart from the framewok

\footnotetext{
Keywords and phrases. Continuous transportation models, traffic congestion.

1 Université Paris Dauphine, CEREMADE, UMR CNRS 7534, Pl. de Lattre de Tassigny, 75775 Paris Cedex 16, France; carlier@ceremade.dauphine.fr

2 Scuola Normale Superiore, Classe di Scienze, Piazza dei Cavalieri 7, 56126, Pisa, Italy; f.santambrogio@sns.it
}

(c) EDP Sciences, SMAI 2005 
of Buttazzo and Santambrogio in our choice of the first two terms. In [5], the transportation cost term is given by a $p$-Wasserstein distance between $\mu$ and $\nu$, whereas, in the present paper, in order to take into account (in a special case) congestion effects, we are lead to consider as a transportation cost the squared norm of $\mu-\nu$ in the dual space of some subspace of $H^{1}(\Omega)$ (see Sect. 2 for details). In dimension 2, this in particular prevents the presence of atoms of $\mu-\nu$. Hence, contrary to [5], who considered a term forcing the distribution of services $\nu$ to be concentrated in at most countably many locations, we rather consider an interaction term of the form:

$$
H(\nu):=\int_{\bar{\Omega} \times \bar{\Omega}} V \mathrm{~d}(\nu \otimes \nu),
$$

where $V(x, y)$ is, for instance, an increasing function of $|x-y|$. Such a term, studied in [9] as well, has already been proposed in [10] as a concentration term useful in similar urban planning problems.

The next section is devoted to modelling traffic congestion. In Section 3, we write down the variational problem and establish existence of minimizers. In Section 4, using the strict convexity of the functional with respect to $\mu$, we characterize the optimal $\mu$ for fixed $\nu$. In Section 5 , after reformulating the initial problem with respect to $\nu$ only, we give necessary optimality conditions. In Section 6, we introduce approximated problems, from which we are able to recover regularity properties of the minimizers. The approximation strategy consists in adding two terms to the functional : a squared $L^{2}$ norm term and a squared 2-Wasserstein distance term. Using elliptic regularity theory and optimal transportation arguments, we obtain $L^{\infty}$ estimates when $\Omega$ is convex and $L_{\text {loc }}^{2}$ estimates in the general case. In Section 7, qualitative properties of the support of optimal $\nu$ 's are established (nonempty interior, simple-connectedness). Finally, in Section 8, we study in details some examples; we first treat the unidimensional case, in which one can use a special displacement convexity property to derive a uniqueness result, then we treat the case when the interaction term is the variance of $\nu$ in two dimensions.

\section{TRAFFIC CONGESTION}

In this section, we formally describe how we model congestion effects in the transportation cost functional. Our analysis builds upon the continuous transportation model of Beckmann (see $[2,3]$ ).

We are given an urban area $\Omega$, which is an open bounded connected subset of $\mathbb{R}^{2}$ satisfying some smoothness assumptions that will be made precise later, and we denote by $\mu$ and $\nu$ the respective distributions of residents and services in the city. As a normalization, we may assume that $\mu$ and $\nu$ are probability measures on $\bar{\Omega}$ and that $\mu$ (respectively $\nu$ ) also gives the distribution of consumption (respectively of production) so that the signed measure $\mu-\nu$ represents the local measure of excess demand. Following [2], we assume that the consumers' traffic is given by a traffic flow field, i.e. a vector field $\mathbf{Y}: \Omega \rightarrow \mathbb{R}^{2}$ whose direction indicates the consumers' travel direction and whose modulus $|\mathbf{Y}|$ is the intensity of traffic.

The relationship between the excess demand and the traffic flow is obtained from an equilibrium condition as follows. There is equilibrium in a subregion $K \subset \Omega$ if the outflow of consumers equals the excess demand of $K$ :

$$
\int_{\partial K} \mathbf{Y} \cdot n \mathrm{~d} \mathcal{H}^{n-1}=(\mu-\nu)(K) .
$$

Since the previous has to hold for arbitrary $K$, this formally yields:

$$
\operatorname{div} \mathbf{Y}=\mu-\nu .
$$

It is also assumed that the urban area is isolated, i.e. no traffic flow should cross the boundary of the city, hence:

$$
\mathbf{Y} \cdot n=0 \text { on } \partial \Omega
$$


If the transportation cost per consumer is assumed to be uniform, then one may define the transportation cost between $\mu$ and $\nu$ as the value of the minimal flow problem:

$$
\inf \left\{\int_{\Omega}|\mathbf{Y}(x)| \mathrm{d} x: \mathbf{Y} \text { satisfies }(2.1)-(2.2)\right\} .
$$

Of course, one generally has to look for generalized (i.e. vector-valued measures) solutions of the previous problem. Let us also mention that the previous problem (or its extension to measures) is tightly connected to the notion of transport density in the Monge-Kantorovich optimal transportation problem (where cost $=$ euclidean distance), and its value coincides with the 1-Wasserstein distance between $\mu$ and $\nu$. We refer to De Pascale and Pratelli [7], for details and very interesting regularity results for transport density in the Monge-Kantorovich problem.

Now, in order to take into account congestion effects, it is more realistic to assume that the transportation cost per consumer at a point $x$ depends on the intensity of traffic at $x$ itself. Let $g: \mathbb{R}_{+} \rightarrow \mathbb{R}_{+}$be a given nondecreasing function, and assume that if the traffic flow is $\mathbf{Y}$ then the transportation cost per consumer at $x$ is $g(|\mathbf{Y}(x)|)$. It is natural, at this point, to define the transportation cost between $\mu$ and $\nu$ as:

$$
C_{g}(\mu, \nu):=\inf \left\{\int_{\Omega} g(|\mathbf{Y}(x)|)|\mathbf{Y}(x)| \mathrm{d} x: \mathbf{Y} \text { satisfies }(2.1)-(2.2)\right\} .
$$

For the sake of simplicity, we will assume, from now on, that $g(t)=t$ for all $t \in \mathbb{R}_{+}$, and define the cost:

$$
C(\mu, \nu):=\inf \left\{\int_{\Omega}|\mathbf{Y}(x)|^{2} \mathrm{~d} x: \mathbf{Y} \text { satisfies }(2.1)-(2.2)\right\},
$$

where (2.1)-(2.2) are understood in the weak sense, hence read as:

$$
\int_{\Omega} \mathbf{Y} \cdot \nabla \phi=\int_{\bar{\Omega}} \phi \mathrm{d}(\mu-\nu), \text { for all } \phi \in C^{1}(\bar{\Omega}) .
$$

Let us define:

$$
X:=\left\{\phi \in H^{1}(\Omega): \int_{\Omega} \phi=0\right\} .
$$

$X$ is a Hilbert space, when equipped with the following inner product and norm:

$$
\langle\phi, \psi\rangle_{X}:=\int_{\Omega} \nabla \phi \cdot \nabla \psi,\|\phi\|_{X}^{2}:=\langle\phi, \phi\rangle_{X} .
$$

As usual, we shall identify $X$ and its dual $X^{\prime}$ by Riesz's isomorphism: for every $f \in X^{\prime}$, there exists, unique, $\phi \in X$ such that:

$$
\langle\phi, \psi\rangle_{X}=f(\psi) \text { for all } \psi \in X .
$$

This implies:

$$
\|f\|_{X^{\prime}}=\|\phi\|_{X}
$$

We shall also write (2.4) in the form:

$$
\begin{cases}-\Delta \phi=f & \text { in } \Omega, \\ \frac{\partial \phi}{\partial n}=0 & \text { on } \partial \Omega, \phi \in X .\end{cases}
$$


With those definitions in mind, it is easy to check that our cost functional given by (2.3) may also be written as:

$$
C(\mu, \nu)= \begin{cases}\|\mu-\nu\|_{X^{\prime}}^{2} & \text { if } \mu-\nu \in X^{\prime} \\ +\infty & \text { otherwise }\end{cases}
$$

Equivalently, we have:

$$
C(\mu, \nu)^{\frac{1}{2}}=\sup \left\{\int_{\bar{\Omega}} \phi \mathrm{d}(\mu-\nu): \phi \in C^{1}(\bar{\Omega}), \int_{\Omega} \phi=0,\|\phi\|_{X} \leq 1\right\} .
$$

\section{The Minimization PROBLEM}

In what follows, $\Omega$ is a bounded and connected open subset of $\mathbb{R}^{2}, V$ is a nonnegative l.s.c. function on $\mathbb{R}^{2}$ and $\mathcal{L}^{2}$ denotes the 2 -dimensional Lebesgue measure on $\Omega$. We consider the variational problem:

$$
\inf \{F(\mu, \nu)=C(\mu, \nu)+G(\mu)+H(\nu): \mu, \nu \text { probabilities on } \bar{\Omega}\}
$$

where:

and

$$
\begin{gathered}
C(\mu, \nu)= \begin{cases}\|\mu-\nu\|_{X^{\prime}}^{2} & \text { if } \mu-\nu \in X^{\prime}, \\
+\infty & \text { otherwise; }\end{cases} \\
G(\mu)= \begin{cases}\int_{\Omega} u^{2} & \text { if } \mu=u \cdot \mathcal{L}^{2}, u \in L^{2}(\Omega), \\
+\infty & \text { otherwise; }\end{cases}
\end{gathered}
$$

$$
H(\nu):=\int_{\bar{\Omega} \times \bar{\Omega}} V(x, y)(\nu \otimes \nu)(\mathrm{d} x, \mathrm{~d} y) .
$$

Theorem 3.1. Assume that $V$ is bounded from below, l.s.c. and there exist probability measures $\mu_{0}$ and $\nu_{0}$ on $\bar{\Omega}$ such that $F\left(\mu_{0}, \nu_{0}\right)<+\infty$. Then the minimization problem (3.1) has at least one solution.

Proof. First it is clear that the infimum of (3.1) is finite. Due to the weak-* compactness of the space of probability measures on $\bar{\Omega}$ (denoted by $\mathcal{P}(\bar{\Omega})$ in the sequel), the existence will directly follow from the weak-* lower semicontinuity of $F$. The weak-* lower semicontinuity of $G$ is clear, that of the interaction functional $H$ is easy to establish and that of $C$ follows from formula (2.7).

\section{Minimization with Respect to $\mu$}

In this paragraph, we consider for a fixed probability $\nu$ (with $\nu \in X^{\prime}$ ) the minimization of $F$ with respect to $\mu$ :

$$
\inf \{C(\mu, \nu)+G(\mu): \mu \text { probability measure on } \bar{\Omega}\} .
$$

Proposition 4.1. Given $\nu \in \mathcal{P}(\bar{\Omega}) \cap X^{\prime}$, then (4.1) admits a unique solution $\mu$ which is characterized by $\mu=\phi \cdot \mathcal{L}^{2}$, where $\phi \in H^{1}(\Omega)$ is the solution of:

$$
\begin{cases}-\Delta \phi+\phi=\nu & \text { in } \Omega, \\ \frac{\partial \phi}{\partial n}=0 & \text { on } \partial \Omega .\end{cases}
$$

Proof. It is obvious that (4.1) admits a unique solution $\mu=u \cdot \mathcal{L}^{2}$ with $u \in L^{2}(\Omega)$. Let $p$ be a probability measure on $\bar{\Omega}$ with $p=v \cdot \mathcal{L}^{2}$ and $v \in L^{2}(\Omega)$ (which implies at once $p \in X^{\prime}$ ). For $\varepsilon \in(0,1)$, one has:

$$
0 \leq C(\mu+\varepsilon(p-\mu), \nu)+G(\mu+\varepsilon(p-\mu))-C(\mu, \nu)-G(\mu) .
$$


Let $\psi \in X$ be the solution of:

Similarly, let $\eta \in X$ be the solution of:

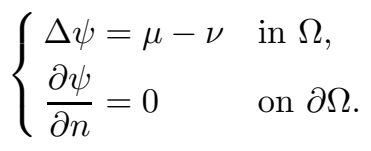

We then have:

$$
\begin{cases}\Delta \eta=p-\mu & \text { in } \Omega \\ \frac{\partial \eta}{\partial n}=0 & \text { on } \partial \Omega\end{cases}
$$

$$
\begin{aligned}
C(\mu+\varepsilon(p-\mu), \nu) & =\|\mu-\nu+\varepsilon(p-\mu)\|_{X^{\prime}}^{2}=\|\psi+\varepsilon \eta\|_{X}^{2} \\
& =C(\mu, \nu)+2 \varepsilon \int_{\Omega} \nabla \psi \cdot \nabla \eta+\varepsilon^{2} \int_{\Omega}|\nabla \eta|^{2} \\
& =C(\mu, \nu)-2 \varepsilon \int_{\Omega} \psi(v-u)+\varepsilon^{2}\|p-\mu\|_{X^{\prime}}^{2}
\end{aligned}
$$

Similarly

$$
G(\mu+\varepsilon(p-\mu))=G(\mu)+2 \varepsilon \int_{\Omega} u(v-u)+\varepsilon^{2} \int_{\Omega}(v-u)^{2} .
$$

Replacing (4.6) and (4.7) in (4.3), dividing by $\varepsilon$ and letting $\varepsilon \rightarrow 0^{+}$, yields:

$$
\int_{\Omega}(v-u)(u-\psi) \geq 0
$$

Since $p=v \cdot \mathcal{L}^{2}$ is an arbitrary probability measure (with $v \in L^{2}(\Omega)$ ), (4.8) can also be written as:

$$
\text { there exists } m \in \mathbb{R} \text { such that: } u-\psi \geq m, u-\psi=m \mu \text {-a.e. }
$$

Since $u \geq 0$, this also implies $u=(\psi+m)_{+}$(as usual, $t_{+}:=\max (0, t), t_{-}:=\max (0,-t)$, for all $\left.t \in \mathbb{R}\right)$.

Define then $\phi:=(\psi+m)$. Let us prove that $\phi \geq 0$ so that we get $u=\phi$. First notice that $\phi_{-}=0 \mu$-a.e. (using (4.9)), and then get:

$$
\int_{\Omega} \nabla \phi \cdot \nabla \phi_{-}=-\int_{\{\phi<0\}}|\nabla \phi|^{2}=\int_{\Omega} \phi_{-} \mathrm{d}(\nu-\mu)=\int_{\Omega} \phi_{-} \mathrm{d} \nu \geq 0 .
$$

This proves $\phi_{-}=0$ and hence $u=\phi$; replacing in (4.4), we get that $\phi$ is the solution of (4.2).

\section{Optimality conditions}

Thanks to Proposition 4.1, we can reformulate the problem (3.1) in terms of $\nu$ only. More precisely, define for every probability measure $\nu$ on $\bar{\Omega}$ :

$$
J(\nu):=\inf \{F(\mu, \nu): \mu \text { probability measure on } \bar{\Omega}\} .
$$

By Proposition 4.1, we have:

$$
J(\nu)= \begin{cases}\int_{\Omega}\left(|\nabla \phi|^{2}+\phi^{2}\right)+H(\nu) & \text { with } \phi \text { the solution of }(4.2) \text { if } \nu \in X^{\prime} \\ +\infty & \text { otherwise. }\end{cases}
$$


Identifying $H^{1}(\Omega)$ and its dual $H^{1}(\Omega)^{\prime}$ via Riesz's isomorphism for its usual Hilbertian structure:

$$
\begin{gathered}
\langle\phi, \psi\rangle_{H^{1}(\Omega)}:=\int_{\Omega}(\nabla \phi \cdot \nabla \psi+\phi \psi), \\
\|\phi\|_{H^{1}(\Omega)}^{2}:=\langle\phi, \phi\rangle_{H^{1}(\Omega)},
\end{gathered}
$$

we may also rewrite $J$ as:

$$
J(\nu)= \begin{cases}\|\nu\|_{H^{1}(\Omega)^{\prime}}^{2}+H(\nu) & \text { if } \nu \in H^{1}(\Omega)^{\prime} \\ +\infty & \text { otherwise. }\end{cases}
$$

Finally, the reformulation of (3.1) reads as:

$$
\inf \{J(\nu): \nu \text { probability measure on } \bar{\Omega}\} .
$$

In what follows, for every $\nu \in H^{1}(\Omega)^{\prime}$, we will say that $\phi \in H^{1}(\Omega)$ is the potential of $\nu$ if:

$$
\langle\phi, \psi\rangle_{H^{1}(\Omega)}=\nu(\psi), \text { for all } \psi \in H^{1}(\Omega) .
$$

Put differently, the potential of $\nu$ is the weak solution of:

$$
\begin{cases}-\Delta \phi+\phi=\nu & \text { in } \Omega, \\ \frac{\partial \phi}{\partial n}=0 & \text { on } \partial \Omega .\end{cases}
$$

Let us also remark that if, in addition, $\nu$ is a probability measure on $\bar{\Omega}$ and $\phi$ its potential, then $\phi \cdot \mathcal{L}^{2}$ is a probability measure on $\bar{\Omega}$ as well.

Let us denote by $\mathcal{C}$ the set of probability measures belonging to the domain of $J$ :

$$
\mathcal{C}=\mathcal{P}(\bar{\Omega}) \cap H^{1}(\Omega)^{\prime}=\left\{\nu \in H^{1}(\Omega)^{\prime}: \nu \geq 0 \text { in } H^{1}(\Omega)^{\prime}, \nu(1)=1\right\} .
$$

In general, the interaction functional $H$ is not convex. However, in the small case, i.e. when either $V$ or $\Omega$ is small (in a sense quantified below) then, due to the term $\|\cdot\|_{H^{1}(\Omega)^{\prime}}^{2}$, the quadratic functional $J$ is in fact strictly convex.

Assume that $V \in C^{2}(\bar{\Omega} \times \bar{\Omega}, \mathbb{R})$ and define:

$$
c_{\Omega, V}:=\left(\int_{\Omega}\left(\|V(x, .)\|_{H^{1}(\Omega)}^{2}+\left\|\partial_{x_{1}} V(x, .)\right\|_{H^{1}(\Omega)}^{2}+\left\|\partial_{x_{2}} V(x, .)\right\|_{H^{1}(\Omega)}^{2}\right) \mathrm{d} x\right)^{\frac{1}{2}} .
$$

Proposition 5.1. Assume that $V \in C^{2}(\bar{\Omega} \times \bar{\Omega}, \mathbb{R})$ and let $c_{\Omega, V}$ be defined by (5.4). If $c_{\Omega, V}<1$, then $J$ is a strictly convex functional on $\mathcal{C}$; (5.1) then admits a unique solution.

Proof. Given $\nu \in \mathcal{C}$, let us define:

$$
T_{\nu}(x):=\nu(V(x, .))=\int_{\bar{\Omega}} V(x, y) \nu(\mathrm{d} y) .
$$

Since $T_{\nu} \in H^{1}(\Omega)$, we have, on the one hand:

$$
|H(\nu)|=\left|\nu\left(T_{\nu}\right)\right| \leq\|\nu\|_{H^{1}(\Omega)^{\prime}}\left\|T_{\nu}\right\|_{H^{1}(\Omega)} .
$$


On the other hand:

$$
\begin{aligned}
T_{\nu}(x)^{2}+\left|\nabla T_{\nu}(x)\right|^{2}=\left(\int_{\bar{\Omega}} V(x, y) \nu(\mathrm{d} y)\right)^{2} & +\left|\int_{\bar{\Omega}} \nabla_{x} V(x, y) \nu(\mathrm{d} y)\right|^{2} \\
& \leq\|\nu\|_{H^{1}(\Omega)^{\prime}}^{2}\left(\|V(x, .)\|_{H^{1}(\Omega)}^{2}+\left\|\partial_{x_{1}} V(x, .)\right\|_{H^{1}(\Omega)}^{2}+\left\|\partial_{x_{2}} V(x, .)\right\|_{H^{1}(\Omega)}^{2}\right) .
\end{aligned}
$$

Integrating the previous inequality and using (5.5), we then get:

$$
|H(\nu)| \leq c_{\Omega, V}\|\nu\|_{H^{1}(\Omega)^{\prime}}^{2},
$$

so that:

$$
J(\nu) \geq\left(1-c_{\Omega, V}\right)\|\nu\|_{H^{1}(\Omega)^{\prime}}^{2}
$$

and the claim of the proposition easily follows using the fact that $J$ is quadratic.

Let $V^{s}$ denote the symmetric part of $V$ :

$$
V^{s}(x, y):=\frac{1}{2}(V(x, y)+V(y, x)) .
$$

The first-order optimality conditions for (5.1) are given by the following result:

Proposition 5.2. Assume that $V \in C^{2}(\bar{\Omega} \times \bar{\Omega}, \mathbb{R})$. Given $\nu \in \mathcal{C}$, let $\phi$ be the potential of $\nu$ and let $T_{\nu}^{s}$ be defined, for all $x \in \Omega$, by:

$$
T_{\nu}^{s}(x):=\nu\left(V^{s}(x, .)\right)=\int_{\bar{\Omega}} V^{s}(x, y) \nu(\mathrm{d} y)
$$

If $\nu$ is a solution of (5.1), then there exists a constant $m$ such that:

$$
\phi+T_{\nu}^{s} \geq m, \phi+T_{\nu}^{s}=m \nu \text {-a.e. }
$$

Proof. Let $p \in \mathcal{C}$, and $\eta \in H^{1}(\Omega)$ be the potential of $p-\nu$. Let $\varepsilon \in(0,1)$; since $\nu$ solves (5.1), we have:

$$
0 \leq J(\nu+\varepsilon(p-\nu))-J(\nu)
$$

We also have:

$$
\| \nu+\varepsilon(p-\nu))\left\|_{H^{1}(\Omega)^{\prime}}^{2}=\right\| \phi+\varepsilon \eta\left\|_{H^{1}(\Omega)}^{2}=J(\nu)+2 \varepsilon \int_{\bar{\Omega}} \phi \mathrm{d}(p-\nu)+\varepsilon^{2}\right\| \eta \|_{H^{1}(\Omega)^{\prime}}^{2} .
$$

Similarly:

$$
H(\nu+\varepsilon(p-\nu))=H(\nu)+2 \varepsilon \int_{\bar{\Omega}} T_{\nu}^{s} \mathrm{~d}(p-\nu)+\varepsilon^{2} \int_{\bar{\Omega} \times \bar{\Omega}} V \mathrm{~d}((p-\nu) \otimes(p-\nu)) .
$$

Replacing in (5.8), dividing by $\varepsilon$ and letting $\varepsilon \rightarrow 0^{+}$yields:

$$
\int_{\bar{\Omega}}\left(\phi+T_{\nu}^{s}\right) \mathrm{d}(p-\nu) \geq 0 .
$$

Since $p \in \mathcal{C}$ is arbitrary in the previous inequality, setting:

$$
m:=\int_{\bar{\Omega}}\left(\phi+T_{\nu}^{s}\right) \mathrm{d} \nu
$$

and using the fact that $\phi+T_{\nu}^{s} \in H^{1}(\Omega)$ we then have (in the $H^{1}(\Omega)$ sense):

$$
\phi+T_{\nu}^{s} \geq m, \phi+T_{\nu}^{s}=m \nu \text {-a.e. }
$$


Remark 5.1. Firstly, let us remark that, thanks to Proposition 5.1, if, in addition, $c_{\Omega, V}<1$, problem (5.1) being strictly convex, condition (5.7) is in fact sufficient and fully characterizes the minimizer. This fact will be used several times in the examples of Section 8. Secondly, it should be noticed that in (5.7) $\nu$ appears only indirectly through its potential and $T_{\nu}^{s}$.

\section{Regularity VIA APPROXIMAtion}

The aim of this section is to get some regularity results on the optimal measure $\nu$ by approximating the minimization problem and then looking for some properties of minimizers passing to the limit.

Before considering the approximation, we need to recall useful results regarding: first, the Wasserstein distance from optimal transport theory and, second, elliptic regularity.

Definition 6.1. For every $p \geq 1$ we call $p$-Wasserstein distance between two probability measures $\mu_{1}$ and $\mu_{2}$ on $\bar{\Omega}$ the quantity

$$
W_{p}\left(\mu_{1}, \mu_{2}\right)=\inf \left(\int_{\bar{\Omega} \times \bar{\Omega}}|x-y|^{p} \lambda(\mathrm{d} x, \mathrm{~d} y)\right)^{1 / p}
$$

where the infimum is taken on all transport plans $\lambda$ between $\mu_{1}$ and $\mu_{2}$, that is on all probability measures $\lambda$ on $\bar{\Omega} \times \bar{\Omega}$ whose marginals $\pi_{\#}^{1} \lambda$ and $\pi_{\#}^{2} \lambda$ coincide with $\mu_{1}$ and $\mu_{2}$ respectively.

To recall some properties of Wasserstein distances we need another definition:

Definition 6.2. We say that a function $\psi$ is $c$-concave with respect to the cost $|x-y|^{p}$ if it can be written in the form

$$
\psi(x)=\inf _{y \in \bar{\Omega}}|x-y|^{p}-\chi(y) .
$$

With the same notation we will also say that $\psi$ is the $c$-transform of $\chi$ (and so a function is $c$-concave if and only if it is the $c$-transform of some function), and write $\psi=\chi^{c}$.

Proposition 6.1. The space $\mathcal{P}(\bar{\Omega})$ equipped with any $p$-Wasserstein distance turns out to be a compact metric space with the same topology as that given by the weak* convergence of measures. Moreover, it holds

$$
W_{p}^{p}\left(\mu_{1}, \mu_{2}\right)=\sup _{\psi c-\text { concave }} \int_{\bar{\Omega}} \psi \mathrm{d} \mu_{1}+\int_{\bar{\Omega}} \psi^{c} \mathrm{~d} \mu_{2}
$$

In our discussion we will make use only of 2 -Wasserstein distance and so we will only deal with $c$-concave functions with respect to $|x-y|^{2}$. Notice that those functions coincide with the functions $\psi$ such that $x \mapsto$ $\psi(x)-x^{2}$ is concave in the usual sense. Moreover, being expressed in terms of an inf, it is clear that they are all $L$-Lipschtiz functions, with $L=2 \operatorname{diam} \Omega$. Just a last notation: the $c$-concave functions realizing the maximum in (6.1) will be referred to as Kantorovich potentials in the transport between $\mu_{1}$ and $\mu_{2}$.

As a second tool we need some result on elliptic regularity theory in the case of Neumann conditions. Precisely, we will use the following.

Proposition 6.2. Consider the elliptic equation (4.2), which is always endowed with an unique solution for every $\nu \in X^{\prime}$. Then it holds:

- if $\Omega$ is an open set with $C^{2}$ boundary and $\nu \in L^{p}(\Omega)$ then $\phi \in W^{2, p}(\Omega)$;

- if $\Omega$ is an open set with $C^{2, \alpha}$ boundary and $\nu \in C^{0, \alpha}(\Omega)$ then $\phi \in C^{2, \alpha}(\Omega)$.

We refer to [1] for both implications. For the Holder theory we can refer also to [8], whose results in Chapter 6 , Section 7, have to be adapted, while for the $L^{p}$ theory in the case $p=2$ the ninth chapter in [4] can be seen as well. From now on, we will call "regular" those open sets whose boundary is $C^{2, \alpha}$ for at least a positive value of $\alpha$. 
In our approximation, we want to retrieve information on all minimizers of our problem (in general, when $J$ is not convex they could be more than one), and so we define some functionals $J_{\varepsilon}$ for every choice of $\bar{\nu} \in \operatorname{argmin} J$.

We set, for small $\varepsilon>0$,

$$
J_{\varepsilon}(\nu)=J(\nu)+\varepsilon W_{2}^{2}\left(\nu, \nu_{\varepsilon}\right)+\delta_{\varepsilon}\|\nu\|_{L^{2}(\Omega)}^{2},
$$

where $\left(\nu_{\varepsilon}\right)_{\varepsilon}$ is a sequence of measures which are absolutely continuous with a strictly positive density, approximating $\bar{\nu}$ in the $W_{2}$ distance, and $\delta_{\varepsilon}$ is a small parameter depending on $\varepsilon$ to be properly chosen.

Since the semicontinuity of the terms we have added with respect to weak* topology is clear, we get the existence of at least a minimizer $\bar{\nu}_{\varepsilon}$ for each functional $J_{\varepsilon}$. We have the following result, which is nothing but an ad hoc modification of general $\Gamma$-convergence concepts.

Proposition 6.3. It is possible to choose the parameters $\delta_{\varepsilon}$ and the sequence $\left(\nu_{\varepsilon}\right)_{\varepsilon}$ in such a way that the sequence of minimizers $\left(\bar{\nu}_{\varepsilon}\right)_{\varepsilon}$ of $J_{\varepsilon}$ tends to $\bar{\nu}$ in the weak* topology (or, equivalently, with respect to the $W_{2}$ distance).

Proof. We choose $\nu_{\varepsilon} \in L^{2}(\Omega)$ such that $J\left(\nu_{\varepsilon}\right) \leq J(\bar{\nu})+\varepsilon^{2} / 2$, and maintaining the fact that $\nu_{\varepsilon} \stackrel{*}{\sim} \bar{\nu}$. This is possible thanks to Lemma 6.4, choosing an $L^{2}$ sequence $\left(\nu_{\varepsilon}\right)_{\varepsilon}$ which approximates $\bar{\nu}$ in the strong topology of $H^{1}(\Omega)^{\prime}$ and noticing that also the interaction term is in fact continuous with respect to this convergence. It is not difficult to choose the densities of the measures $\nu_{\varepsilon}$ to be positive as required. Then we set $\delta_{\varepsilon}=\varepsilon^{2}\left(\left\|\nu_{\varepsilon}\right\|_{L^{2}(\Omega)}\right)^{-2} / 2$.

So we have

$$
J\left(\bar{\nu}_{\varepsilon}\right)+\varepsilon W_{2}^{2}\left(\bar{\nu}_{\varepsilon}, \nu_{\varepsilon}\right)+\delta_{\varepsilon}\left\|\bar{\nu}_{\varepsilon}\right\|_{L^{2}(\Omega)}^{2} \leq J\left(\nu_{\varepsilon}\right)+\delta_{\varepsilon}\left\|\nu_{\varepsilon}\right\|_{L^{2}(\Omega)}^{2} \leq J(\bar{\nu})+\varepsilon^{2} .
$$

Since $\bar{\nu}$ is a minimizer for $J$ we have $J\left(\bar{\nu}_{\varepsilon}\right) \geq J(\bar{\nu})$, and so we get

$$
J(\bar{\nu})+\varepsilon W_{2}^{2}\left(\bar{\nu}_{\varepsilon}, \nu_{\varepsilon}\right) \leq J(\bar{\nu})+\varepsilon^{2},
$$

where we have neglected the positive term $\delta_{\varepsilon}\left\|\bar{\nu}_{\varepsilon}\right\|_{L^{2}(\Omega)}^{2}$. By simplifying and dividing by $\varepsilon$ we get

$$
W_{2}^{2}\left(\bar{\nu}_{\varepsilon}, \nu_{\varepsilon}\right) \leq \varepsilon,
$$

and so

which is the thesis.

$$
W_{2}\left(\bar{\nu}_{\varepsilon}, \bar{\nu}\right) \leq \sqrt{\varepsilon}+W_{2}\left(\bar{\nu}, \nu_{\varepsilon}\right) \rightarrow 0,
$$

Lemma 6.4. The subspace $C_{c}^{\infty}(\Omega) \subset L^{2}(\Omega)$ is dense in the Hilbert space $H^{1}(\Omega)^{\prime}$.

Proof. It is sufficient to show the following implication:

$$
\xi \in H^{1}(\Omega)^{\prime},\langle\xi, f\rangle_{H^{1}(\Omega)^{\prime}}=0 \text { for all } f \in C_{c}^{\infty}(\Omega) \Rightarrow \xi=0 .
$$

After calling $\psi_{\xi}$ and $\psi_{f}$ the potentials of $\xi$ and $f$, respectively, we have

$$
\langle\xi, f\rangle_{H^{1}(\Omega)^{\prime}}=\left\langle\psi_{\xi}, \psi_{f}\right\rangle_{H^{1}(\Omega)}=\int_{\Omega} \psi_{\xi} \psi_{f}+\int_{\Omega} \nabla \psi_{\xi} \cdot \nabla \psi_{f}=\int_{\Omega} \psi_{\xi} f .
$$

Consequently, the condition of being $\xi$ orthogonal to every $f \in C_{c}^{\infty}(\Omega)$ in $H^{1}(\Omega)^{\prime}$ implies that the potential of $\xi$ must be orthogonal in $L^{2}(\Omega)$ to all $C_{c}^{\infty}$ functions. So $\psi_{\xi}$ must be identically 0 and then $\xi=0$.

Having established the convergence of the minimizers $\bar{\nu}_{\varepsilon}$ to $\bar{\nu}$, we look for uniform estimates of such minimizers. From now on, we will make use of the following assumption on the function $V$ :

Vdiod ( $V$ depends increasingly on distances): $V$ is a function of the form $V(x, y)=v\left(|x-y|^{2}\right)$ for a $C^{2}$ strictly increasing function $v$ with $v^{\prime}(s)>0$ for $s>0$.

Obviously, under this hypothesis, $V$ is a symmetric function and so $V=V^{s}$ and $T_{\nu}=T_{\nu}^{s}$ for every probability measure $\nu$. 


\section{1. $\boldsymbol{L}^{\infty}$ estimates in the convex case}

Theorem 6.5. Suppose that $\Omega$ is a bounded, regular and strictly convex open subset of $\mathbb{R}^{2}$ and that $\mathbf{V d i o d}$ holds. Then, every minimizer $\bar{\nu}_{\varepsilon}$ of $J_{\varepsilon}$ is an absolutely continuous measure with $L^{\infty}$ density, bounded by a uniform constant depending on $\Omega$ and on $\|V\|_{C^{2}(\Omega)}$. Consequently, $\bar{\nu}$ has a density bounded by the same constant as well.

Proof. We write down a necessary optimality condition on $\bar{\nu}_{\varepsilon}$. To obtain it, we act as in the proof of Proposition 5.2. We have only to consider two additional terms. The $L^{2}$ term is easy to deal with: if we set $\nu_{\varepsilon, t}=\nu_{\varepsilon}+t\left(p-\nu_{\varepsilon}\right)$ for small $\varepsilon, t \in[0,1]$ and an arbitrary probability $p \in L^{2}(\Omega)$, we have

$$
\lim _{t \rightarrow 0} \frac{\left\|\nu_{\varepsilon, t}\right\|_{L^{2}(\Omega)}^{2}-\left\|\bar{\nu}_{\varepsilon}\right\|_{L^{2}(\Omega)}^{2}}{t}=2 \int_{\Omega}\left(p-\bar{\nu}_{\varepsilon}\right) \bar{\nu}_{\varepsilon} .
$$

For the Wasserstein term, we behave as in [5]. Let us choose for each $t$ a Kantorovich potential $\psi_{\varepsilon, t}$ for the transport between $\nu_{\varepsilon, t}$ and $\nu_{\varepsilon}$, and let $\psi_{\varepsilon}$ be the only Kantorovich potential (up to additive constants) between $\bar{\nu}_{\varepsilon}$ and $\nu_{\varepsilon}$ (uniqueness comes from the fact that the density of $\nu_{\varepsilon}$ is positive everywhere on the connected open set $\Omega$ ). We can choose all these Kantorovich potentials to vanish on a same point. Remember that they are all $L$-Lipschitz functions. We then have

$$
W_{2}^{2}\left(\nu_{\varepsilon, t}, \nu_{\varepsilon}\right)-W_{2}^{2}\left(\bar{\nu}_{\varepsilon}, \nu_{\varepsilon}\right) \leq t \int_{\Omega} \psi_{\varepsilon, t} \mathrm{~d}\left(p-\bar{\nu}_{\varepsilon}\right)
$$

and so

$$
\limsup _{t \rightarrow 0} \frac{W_{2}^{2}\left(\nu_{\varepsilon, t}, \nu_{\varepsilon}\right)-W_{2}^{2}\left(\bar{\nu}_{\varepsilon}, \nu_{\varepsilon}\right)}{t} \leq \int_{\Omega} \psi_{\varepsilon} \mathrm{d}\left(p-\bar{\nu}_{\varepsilon}\right) .
$$

We have used the fact that, up to subsequences, the sequence $\left(\psi_{\varepsilon, t}\right)_{t}$ has a limit and such a limit must be a Kantorovich potential between $\bar{\nu}_{\varepsilon}$ and $\nu_{\varepsilon}$, and so it must be $\psi_{\varepsilon}$.

By this considerations and the same technique as in Proposition 5.2, we get

$$
\begin{aligned}
& \delta_{\varepsilon} \bar{\nu}_{\varepsilon}+\frac{\varepsilon}{2} \psi_{\varepsilon}+\phi_{\varepsilon}+T_{\bar{\nu}_{\varepsilon}} \geq c_{\varepsilon} \text { in } \Omega \\
& \delta_{\varepsilon} \bar{\nu}_{\varepsilon}+\frac{\varepsilon}{2} \psi_{\varepsilon}+\phi_{\varepsilon}+T_{\bar{\nu}_{\varepsilon}}=c_{\varepsilon} \text { for } \bar{\nu}_{\varepsilon}-\text { a.e. } x \in \Omega .
\end{aligned}
$$

Here $\phi_{\varepsilon}$ is the potential of $\bar{\nu}_{\varepsilon}$, and we have identified $\bar{\nu}_{\varepsilon}$ with its density (obviously $\bar{\nu}_{\varepsilon} \in L^{2}(\Omega)$ ). We may write

$$
\bar{\nu}_{\varepsilon}=\frac{1}{\delta_{\varepsilon}}\left(c_{\varepsilon}-\phi_{\varepsilon}-T_{\bar{\nu}_{\varepsilon}}-\frac{\varepsilon}{2} \psi_{\varepsilon}\right)_{+} .
$$

Since $\bar{\nu}_{\varepsilon}$ is $L^{2}$ we have $\phi_{\varepsilon} \in H^{2}(\Omega) \subset C^{0, \alpha}(\Omega)$, and this shows that $\bar{\nu}_{\varepsilon}$ is Holder continuous, since all the functions appearing in the positive part are at least Holder continuous. Consequently, since $\bar{\nu}_{\varepsilon} \in C^{0, \alpha}(\Omega)$, the function $\phi_{\varepsilon}$ turns out to be a $C^{2, \alpha}$ function.

We look for a maximum point $x_{\varepsilon}$ of $\bar{\nu}_{\varepsilon}$ : in it we have a local minimum of the sum $\frac{\varepsilon}{2} \psi_{\varepsilon}+\phi_{\varepsilon}+T_{\bar{\nu}_{\varepsilon}}$. Thanks to $x \mapsto \psi_{\varepsilon}(x)-x^{2}$ being concave, we may write $\psi_{\varepsilon} \leq l+Q$, with equality in $x_{\varepsilon}$, where $l$ is an affine function and $Q(x)=x^{2}$. Consequently $x_{\varepsilon}$ is a local minimum for the sum $\frac{\varepsilon}{2}(l+Q)+\phi_{\varepsilon}+T_{\bar{\nu}_{\varepsilon}}$. Lemma 6.6 shows that $x_{\varepsilon}$ does not belong to the boundary of $\Omega$, at least for small $\varepsilon$. So, since $x_{\varepsilon}$ is an interior point and all the functions involved are at least twice differentiable, we may write, taking the laplacians,

$$
0 \leq 2 \varepsilon+\Delta \phi_{\varepsilon}\left(x_{\varepsilon}\right)+\Delta T_{\bar{\nu}_{\varepsilon}}\left(x_{\varepsilon}\right) .
$$

In this case we can use $\bar{\nu}_{\varepsilon}=\phi_{\varepsilon}-\Delta \phi_{\varepsilon}$ to estimate $\bar{\nu}_{\varepsilon}\left(x_{\varepsilon}\right)$. In fact in $x_{\varepsilon}$ we have $\bar{\nu}_{\varepsilon}\left(x_{\varepsilon}\right)>0$ and so

$$
\phi_{\varepsilon}\left(x_{\varepsilon}\right)-M \leq c_{\varepsilon},
$$


where $M=\sup \left|\psi_{\varepsilon}\right|+\left|T_{\bar{\nu}_{\varepsilon}}\right|$ can be uniformly estimated, $\psi_{\varepsilon}$ being $L$-Lipschitz functions vanishing at a given point in $\Omega$ and $\left|T_{\nu}\right| \leq \sup |V|$ for every probability $\nu$. So it is sufficient to estimate $c_{\varepsilon}$. To do this we can integrate (6.4), obtaining

$$
\delta_{\varepsilon} \geq c_{\varepsilon}|\Omega|-M|\Omega|-1
$$

where we used the fact that both $\bar{\nu}_{\varepsilon}$ and $\phi_{\varepsilon}$ are probability measures. Putting together (6.6) and (6.7) we get $\phi_{\varepsilon} \leq C$, being $C$ a constant depending on $\Omega$ and $\sup V$, and so, by recalling the equality $\bar{\nu}_{\varepsilon}=\phi_{\varepsilon}-\Delta \phi_{\varepsilon}$ and the inequality (6.5) we get

$$
\bar{\nu}_{\varepsilon}\left(x_{\varepsilon}\right) \leq C+2 \varepsilon+\|V\|_{C^{2}(\Omega)} .
$$

Since $x_{\varepsilon}$ is a maximum point we have got an $L^{\infty}$ estimate on $\bar{\nu}_{\varepsilon}$.

Lemma 6.6. Suppose that $\Omega$ is a bounded, regular and strictly convex open subset of $\mathbb{R}^{2}$ and that Vdiod holds. Then, at least for small $\varepsilon$ we have $x_{\varepsilon} \in \Omega$.

Proof. Suppose, on the contrary, to have a sequence $\left(x_{\varepsilon}\right)_{\varepsilon}$ contained in the boundary $\partial \Omega$. Such $x_{\varepsilon}$ is a local minimum point for $\frac{\varepsilon}{2} \psi_{\varepsilon}+\phi_{\varepsilon}+T_{\bar{\nu}_{\varepsilon}}$. In a local minimum point on the boundary the normal exterior derivative should be non positive. The derivative of $\psi_{\varepsilon}$ may also not exist, but we may use the fact that $\psi_{\varepsilon}$ is an $L$-Lipschitz function. Being multiplied by $\varepsilon$, and vanishing by definition the normal derivative of $\phi_{\varepsilon}$, it is not difficult to check that we should have

$$
\limsup _{\varepsilon \rightarrow 0} \frac{\partial T_{\bar{\nu}_{\varepsilon}}}{\partial n}\left(x_{\varepsilon}\right) \leq 0
$$

On the other hand, we have

$$
\frac{\partial T_{\bar{\nu}_{\varepsilon}}}{\partial n}\left(x_{\varepsilon}\right)=2 \int_{\Omega} v^{\prime}\left(\left|x_{\varepsilon}-y\right|^{2}\right)\left(x_{\varepsilon}-y\right) \cdot n\left(x_{\varepsilon}\right) \bar{\nu}_{\varepsilon}(\mathrm{d} y) \geq a_{\delta} \delta \bar{\nu}_{\varepsilon}\left(\Omega \backslash S_{\delta}\left(x_{\varepsilon}\right)\right),
$$

where $a_{\delta}$ is the minimum value of $v^{\prime}$ on $\left[\delta^{2}, \operatorname{diam} \Omega^{2}\right]$ and, for every point $x \in \partial \Omega$, we define $S_{\delta}(x)=$ $\{y \in \Omega \mid(x-y) \cdot n(x) \leq \delta\}$. Condition (6.8) implies that, for every $\delta>0$, it holds $\bar{\nu}_{\varepsilon}\left(\Omega \backslash S_{\delta}\left(x_{\varepsilon}\right)\right) \rightarrow 0$. Taking a limit point $x_{0}$ of the sequence $\left(x_{\varepsilon}\right)_{\varepsilon}$, we will show that this implies that the measure which is the limit of the sequence $\left(\bar{\nu}_{\varepsilon}\right)_{\varepsilon}$ is concentrated on $x_{0}$. This is impossible, since this limit measure is $\bar{\nu}$, which is optimal for $J$, and so it belongs to $H^{1}(\Omega)^{\prime}$. Yet in two dimensions a measure concentrated on a single point does not belong to such a space. To conclude, it is then sufficient to show that, for each ball $\overline{B\left(x_{0}, r\right)}$, it holds $S_{\delta}\left(x_{\varepsilon}\right) \subset \overline{B\left(x_{0}, r\right)}$ for sufficiently small $\delta$ and $\varepsilon$, thus getting $\lim _{\varepsilon} \bar{\nu}_{\varepsilon}\left(\overline{B\left(x_{0}, r\right)}\right)=1$ for every $r>0$. If not, we would have a sequence $\left(y_{\delta, \varepsilon}\right)_{\delta, \varepsilon}$ such that $\left(x_{\varepsilon}-y_{\delta, \varepsilon}\right) \cdot n\left(x_{\varepsilon}\right)<\delta$ and $\left|x-y_{\delta, \varepsilon}\right|>r$. At the limit we get a point $y \in \partial \Omega$ such that $\left(x_{0}-y\right) \cdot n\left(x_{0}\right) \leq 0$ (which implies, in a strictly convex $\Omega, x_{0}=y$ ), but $\left|x_{0}-y\right| \geq r$, and this is absurd.

Remark 6.1. If we want to consider the one-dimensional case (with $\Omega$ an interval) the proof of Lemma 6.6 has to be modified: it is sufficient to say that a measure concentrated at a single point, which is a terminal point of the interval, cannot be optimal. The potential of such a measure can be explicitely computed, being an exponential function, and it can be proven that the optimality condition of Proposition 5.2 cannot hold, at least under the additional assumption $v^{\prime}(0)=0$ in Vdiod.

We conclude this part of the section by a consideration on the consequences of this result on the regularity of the potential $\phi$.

Corollary 6.7. The potential $\phi$ of an optimal measure is a $W^{2, p}$ function for any $1 \leq p<+\infty$ and then a $C^{1, \alpha}$ function too.

Proof. Just apply Proposition 6.2 and consider that $\bar{\nu} \in L^{\infty}(\Omega) \subset L^{p}(\Omega)$ for any finite $p$. The second part of the statement is just a consequence of well-known embedding theorems. 


\subsection{Interior $\boldsymbol{L}^{\mathbf{2}}$ estimates in the general case}

In this section, we look for weaker estimates which are valid in the case of a non convex domain $\Omega$. Let us write $\partial \Omega=\Gamma_{1} \cup \Gamma_{2}$, where $\Gamma_{1}=\partial \Omega \cap \partial(\operatorname{co} \Omega)$ and $\Gamma_{2}=\partial \Omega \backslash \partial(\operatorname{co} \Omega)$.

Theorem 6.8. Suppose that $\Gamma_{1}$ is a strictly convex regular boundary and that Vdiod holds. Then, given a Lipschitz function $\theta$ such that $\mathrm{d}\left(\operatorname{spt} \theta, \Gamma_{2}\right)>0$, the sequence of functions $\left(\theta \bar{\nu}_{\varepsilon}\right)_{\varepsilon}$ is bounded in $L^{2}$.

Proof. We start by testing equation (4.2) for $\bar{\nu}_{\varepsilon}$ against the function $\theta^{2} \bar{\nu}_{\varepsilon}$ :

$$
\int_{\Omega} \bar{\nu}_{\varepsilon}^{2} \theta^{2}=\int_{\Omega} \phi_{\varepsilon} \theta^{2} \bar{\nu}_{\varepsilon}+\int_{\Omega} \nabla \phi_{\varepsilon} \cdot \nabla\left(\theta^{2} \bar{\nu}_{\varepsilon}\right) .
$$

Since on spt $\bar{\nu}_{\varepsilon}$ we have $\nabla \phi_{\varepsilon}=-\delta_{\varepsilon} \nabla \bar{\nu}_{\varepsilon}-\nabla\left(\frac{\varepsilon}{2} \psi_{\varepsilon}+T_{\bar{\nu}_{\varepsilon}}\right)$, we get

$$
\begin{aligned}
\int_{\Omega} \bar{\nu}_{\varepsilon}^{2} \theta^{2}= & \int_{\Omega} \phi_{\varepsilon} \theta^{2} \bar{\nu}_{\varepsilon}-\delta_{\varepsilon} \int_{\Omega} \nabla \bar{\nu}_{\varepsilon} \cdot \nabla\left(\theta^{2} \bar{\nu}_{\varepsilon}\right)-\int_{\Omega} \nabla\left(\frac{\varepsilon}{2} \psi_{\varepsilon}+T_{\bar{\nu}_{\varepsilon}}\right) \cdot \nabla\left(\theta^{2} \bar{\nu}_{\varepsilon}\right) \\
\leq & \int_{\Omega} \phi_{\varepsilon} \theta^{2} \bar{\nu}_{\varepsilon}-\delta_{\varepsilon} \int_{\Omega} \theta^{2}\left|\nabla \bar{\nu}_{\varepsilon}\right|^{2}-2 \delta_{\varepsilon} \int_{\Omega} \bar{\nu}_{\varepsilon} \theta \nabla \bar{\nu}_{\varepsilon} \cdot \nabla \theta \\
& +\int_{\Omega} \Delta\left(\frac{\varepsilon}{2} \psi_{\varepsilon}+T_{\bar{\nu}_{\varepsilon}}\right) \theta^{2} \bar{\nu}_{\varepsilon}-\int_{\partial \Omega} \theta^{2} \bar{\nu}_{\varepsilon}\left(\frac{\partial T_{\bar{\nu}_{\varepsilon}}}{\partial n}-\frac{\varepsilon}{2} L\right) .
\end{aligned}
$$

Using once more $\delta_{\varepsilon} \nabla \bar{\nu}_{\varepsilon}=-\nabla \phi_{\varepsilon}-\nabla\left(\frac{\varepsilon}{2} \psi_{\varepsilon}+T_{\bar{\nu}_{\varepsilon}}\right)$ on spt $\bar{\nu}_{\varepsilon}$ in (6.10), we get:

$$
\begin{aligned}
\int_{\Omega} \bar{\nu}_{\varepsilon}^{2} \theta^{2} \leq \int_{\Omega} \phi_{\varepsilon} \theta^{2} \bar{\nu}_{\varepsilon}-\delta_{\varepsilon} \int_{\Omega} \theta^{2}\left|\nabla \bar{\nu}_{\varepsilon}\right|^{2}+2 \int_{\Omega} \bar{\nu}_{\varepsilon} \theta \nabla \phi_{\varepsilon} \cdot \nabla \theta \\
\quad+2 \int_{\Omega} \bar{\nu}_{\varepsilon} \theta \nabla\left(\frac{\varepsilon}{2} \psi_{\varepsilon}+T_{\bar{\nu}_{\varepsilon}}\right) \cdot \nabla \theta+\int_{\Omega} \Delta\left(\frac{\varepsilon}{2} \psi_{\varepsilon}+T_{\bar{\nu}_{\varepsilon}}\right) \theta^{2} \bar{\nu}_{\varepsilon}-\int_{\partial \Omega} \theta^{2} \bar{\nu}_{\varepsilon}\left(\frac{\partial T_{\bar{\nu}_{\varepsilon}}}{\partial n}-\frac{\varepsilon}{2} L\right) .
\end{aligned}
$$

Notice that the Laplacian appearing in the fifth term is composed by two parts: the Laplacian of a $C^{2}$ function and the Laplacian of a concave one, which is a negative measure. We have six terms that must be estimated:

- the first one is bounded by $\left\|\theta \bar{\nu}_{\varepsilon}\right\|_{L^{2}(\Omega)}\left\|\theta \phi_{\varepsilon}\right\|_{L^{2}(\Omega)}$;

- the second is negative;

- the third is bounded by $\left\|\nabla \phi_{\varepsilon}\right\|_{L^{2}(\Omega)}\left\|\theta \bar{\nu}_{\varepsilon}\right\|_{L^{2}(\Omega)} \operatorname{lip} \theta$;

- the fourth by $\left\|\theta \bar{\nu}_{\varepsilon}\right\|_{L^{2}(\Omega)}\left(\frac{\varepsilon}{2} L+\operatorname{lip} V\right) \operatorname{lip} \theta$;

- the fifth by $\left(2 \varepsilon+\|V\|_{C^{2}(\Omega)}\right)\left\|\theta^{2}\right\|_{L^{\infty}(\Omega)}$;

- the last one is negative for small $\varepsilon$ and it can be proven exactly as in the proof of Lemma 6.6.

The proof is then achieved, since the sequence $\left(\phi_{\varepsilon}\right)_{\varepsilon}$ is bounded in $H^{1}(\Omega)$, thanks to $\left\|\phi_{\varepsilon}\right\|_{L^{2}(\Omega)}^{2}+\left\|\nabla \phi_{\varepsilon}\right\|_{L^{2}(\Omega)}^{2} \leq$ $J_{\varepsilon}\left(\bar{\nu}_{\varepsilon}\right)$. Moreover, the left hand side in (6.11) is quadratic in $\left\|\theta \bar{\nu}_{\varepsilon}\right\|_{L^{2}(\Omega)}$ and the right hand side at most linear, which gives the estimate we were looking for.

Theorem 6.8 gives a local $L^{2}$ bound on the densities $\bar{\nu}_{\varepsilon}$ : this enables us, together with the optimality conditions of Section 5 , to state a stronger regularity result.

Theorem 6.9. Suppose that $\Gamma_{1}$ is a strictly convex regular boundary and that Vdiod holds. Then any optimal measure $\bar{\nu}$ for $J$ can be expressed as $\bar{\nu}=\bar{\nu}^{a}+\bar{\nu}^{s}$, with $\bar{\nu}^{a} \in L^{\infty}(\Omega)$ and $\bar{\nu}^{s}$ a singular measure supported on $\bar{\Gamma}_{2}$.

Proof. By Theorem 6.8 we get that $\bar{\nu}$ is locally $L^{2}$ in $\Omega \backslash \bar{\Gamma}_{2}$. This means, by interior elliptic regularity theory, that its potential $\phi$ is locally $H^{2}$ in the same set, and thus continuous. Hence the equality $\phi=c-T_{\bar{\nu}}$ given by optimality conditions holds true on the whole spt $\bar{\nu}$. So the following holds

$$
\phi I_{\mathrm{spt} \bar{\nu}}=\left(c-T_{\bar{\nu}}\right) I_{\mathrm{spt} \bar{\nu}} \in L^{\infty}(\Omega) .
$$


Moreover, $\mathcal{L}^{2}$-almost everywhere on spt $\bar{\nu}$, we also have $\Delta \phi=-\Delta T_{\bar{\nu}}$ and so

$$
\Delta \phi I_{\mathrm{spt} \bar{\nu}}=-\Delta T_{\bar{\nu}} I_{\mathrm{spt} \bar{\nu}} \in L^{\infty}(\Omega) .
$$

(6.12) and (6.13) together, imply

$$
\bar{\nu} I_{A}=(\phi-\Delta \phi) I_{\mathrm{spt}} \bar{\nu} \in L^{\infty}(\Omega)
$$

where $A=\bar{\Omega} \backslash \Gamma_{2}$. Finally, set $\nu^{a}=\bar{\nu} I_{A}$ and $\nu^{s}=\bar{\nu} I_{\Gamma_{2}}$.

Remark 6.2. In Section 8, an example will be given to show that it is in fact possible that an optimal $\nu$ gives a positive mass to $\Gamma_{2}$

\section{Qualitative Properties of the Minimizers}

In this section, we give some qualitative properties regarding the support of an optimal measure $\nu$. This turns out to be very important, thanks to the following result. In all the section $\Omega$ will be strictly convex, regular, and condition Vdiod will hold.

Proposition 7.1. The $L^{\infty}$ density of any optimal measure $\nu$ coincides almost everywhere in spt $\nu$ with a continuous function.

Proof. Thanks to the regularity of the potential $\phi$ we may say that the equality $\phi=c-T_{\nu}$ holds eveywhere in the support and that, for the laplacian of $\phi$, which is an $L^{p}$ function, it holds $\Delta \phi=-\Delta T_{\nu}$ a.e. From $\nu=\phi-\Delta \phi$ and $V \in C^{2}(\Omega)$, which implies $T_{\nu} \in C^{2}(\Omega)$, we get the thesis.

As a consequence of the previous result, we may say that the reason for possible irregular behaviour of $\nu$ must be traced back to the shape of its support. As far as this shape is concerned, we can only give two general results, whose statements are quite weak.

Proposition 7.2. Suppose, other than the general assumptions of the section, that $V$ is strictly convex. Then the support of $\nu$ has non-empty interior.

Proof. We will show that spt $\nu$ contains a small ball around the point $x_{0}$ defined by $x_{0}=\operatorname{argmin} T_{\nu}$. The function $T_{\nu}$ inherits strict convexity from $V$, and so there exist just one minimizer and just one critical point for $T_{\nu}$. We start by saying that, under the assumption of Theorem 6.5 , we must have spt $\nu \cap \partial \Omega=\emptyset$. Indeed, $\phi$ being a $C^{1, \alpha}$ function, it holds $\nabla \phi=-\nabla T_{\nu}$ on the whole support and this, by calculating $\nabla T_{\nu}$ as in Lemma 6.6 , would otherwise prevent the normal derivative of $\phi$ from vanishing on $\partial \Omega$.

We now want to show that $x_{0} \in \operatorname{spt} \nu$ : to do this consider a maximum point $\bar{x}$ for $\phi$. Such a point must be placed in spt $\nu$, since outside it holds $\Delta \phi=\phi$ and on an interior maximum point we should have a strictly positive value for $\phi$. The same consideration can be performed on the boundary, since we already know that the normal derivative vanishes and no maximum point on the boundary with vanishing normal derivative and positive laplacian is allowed. Notice that outside spt $\nu$ the function $\phi$ is an analytic function because of standard elliptic regularity theory and so it makes sense to consider its laplacian on $\partial \Omega$ too.

Now, it must hold

$$
0=\nabla \phi(\bar{x})=-\nabla T_{\nu}(\bar{x}),
$$

and so $\bar{x}=x_{0}$. Consequently $x_{0}$ is a point in $\operatorname{spt} \nu$ and then in $\Omega$.

Let us now consider for a fixed small value of $\varepsilon>0$ and for $\delta$ in a ball near $0 \in \mathbb{R}^{2}$ the functions

$$
f_{\varepsilon, \delta}(x)=\phi(x)-\frac{\varepsilon}{2}\left|x-\left(x_{0}+\delta\right)\right|^{2} .
$$

The parameter $\varepsilon$ has to be chosen in such a way that in any maximum point of $f_{\varepsilon, \delta}$ it holds $\phi>\varepsilon$ (it is in fact sufficient to satisfy the inequality $\left.|\Omega|^{-1}>\varepsilon\left(1+(\operatorname{diam} \Omega)^{2} / 2\right)\right)$. After choosing $\varepsilon>0$ in such a way, we will think of it as a fixed parameter. 
Now consider $x_{\delta} \in \operatorname{argmin} f_{\varepsilon, \delta}$. Such a point cannot lie on the boundary because of the sign of the normal derivative, and it cannot be outside spt $\nu$, by computing the laplacian. So $x_{\delta} \in \operatorname{spt} \nu$. The point $x_{\delta}$ is characterized by

$$
\left[\varepsilon I d+\nabla T_{\nu}\right]\left(x_{\delta}\right)=\varepsilon\left(x_{0}+\delta\right)
$$

the application on the left hand side being injective since it is monotone (in the usual sense for vector-valued maps). If $\delta=0$ the solution is given by $x_{\delta}=x_{0}$, and so, by standard local inversion theorems, the set of points $x_{\delta}$ covers a small ball around $x_{0}$. Such a ball must consequently be contained in $\operatorname{spt} \nu$.

Our next result deals with the topology of the support

Proposition 7.3. Suppose, other than the general assumptions of the section, that $V$ is strictly subharmonic, i.e. $\Delta V>0$. Then the support of $\nu$ is simply connected, in the sense that, if $\omega \subset \Omega$ is an open set such that $\partial \omega \subset \operatorname{spt} \nu$, then $\omega \subset \operatorname{spt} \nu$.

Proof. We consider a maximum point $x_{0}$ for $\phi+T_{\nu}$ in $\bar{\omega}$. Let us recall that $\phi=c-T_{\nu}$ in spt $\nu$ and $\phi \geq c-T_{\nu}$ everywhere. So, if the maximum point belongs to $\operatorname{spt} \nu$, we have $\phi=c-T_{\nu}$ on $\omega$. On the other hand, it is impossible to have $x_{0} \in \omega \backslash \operatorname{spt} \nu$ because there we have $\Delta\left(\phi+T_{\nu}\right)=\phi+\Delta T_{\nu}>\phi \geq 0$, since $T_{\nu}$ inherits strict subharmonicity from $V$. Consequently, $x_{0}$ must belong to $\bar{\omega} \cap \operatorname{spt} \nu$. Then we have $\phi=c-T_{\nu}$ and so $\Delta \phi=-\Delta T_{\nu}$ in the whole $\omega$ and so

$$
\nu=\phi+\Delta T_{\nu}>\phi \geq 0 \text { in } \omega,
$$

which obviously implies $\omega \subset \operatorname{spt} \nu$.

\section{EXAMPLES}

\subsection{The unidimensional case}

It's worthwhile to consider the case where $\Omega=(-R, R)$ is a bounded interval in $\mathbb{R}$, instead of a twodimensional open set. Obviously from the point of view of applications it sounds less interesting, even if sometimes in urban economics unidimensional models have been used to deal with the case of very long and narrow cities (and in fact some towns on the sea shore are not far from being one dimensional). From a mathematical point of view, the main interest lies in the fact that we can show the functional $J$ to be displacement convex (or strictly displacement convex), under convexity assumption on $V$. This gives uniqueness of the minimizer, but it is also important since displacement convexity has never been studied for functionals of the form of the squared $\left(H^{1}\right)^{\prime}$ norm. Anyway, the techniques here used to get this term geodesically convex are very specific to the unidimensional case . $[9]$.

Let us recall the notion of displacement convexity, which has been introduced and developed by McCann in

Definition 8.1. Let $\mu$ and $\nu$ be two probability measures on $\bar{\Omega}$ and let $T: \bar{\Omega} \rightarrow \bar{\Omega}$ be an optimal transport map (unique if $\mu$ is absolutely continuous) between them with respect to the cost function $|x-y|^{2}$. We consider the curve $\gamma^{T}$ with values in $\mathcal{P}(\bar{\Omega})$ endowed with the 2 -Wasserstein distance, given by

$$
t \mapsto \gamma^{T}(t)=[(1-t) I d+t T]_{\#} \mu .
$$

A functional $F$ defined on $\mathcal{P}(\bar{\Omega})$ is said to be displacement convex if all the maps $t \mapsto F\left(\gamma^{T}(t)\right)$ are convex on $[0,1]$ for every choice of $\mu, \nu$ and $T$.

Before presenting the displacement convexity result, we need to recall the concept of Green function and its link to the squared $\left(H^{1}\right)^{\prime}$ norm. The following result can be adapted to any dimension.

Proposition 8.1. For every measure $\nu \in H^{1}(\Omega)^{\prime}$ it holds

$$
\|\nu\|_{H^{1}(\Omega)^{\prime}}^{2}=\int_{\Omega \times \Omega} G(x, y) \nu(\mathrm{d} x) \nu(\mathrm{d} y)
$$


the function $G_{x}=G(x, \cdot)$ being for every $x \in \Omega$ the solution to

$$
\begin{cases}-\Delta_{y} G_{x}+G_{x}=\delta_{x} & \text { in } \Omega, \\ \frac{\partial G_{x}}{\partial n}=0 & \text { on } \partial \Omega\end{cases}
$$

i.e. $G$ is the Green function for the operator $-\Delta+I d$ with Neumann boundary conditions.

Proof. First, we notice that it holds

$$
\|\nu\|_{H^{1}(\Omega)^{\prime}}^{2}=\int_{\Omega} \phi^{2}+\int_{\Omega}|\nabla \phi|^{2}=\int_{\Omega} \phi \mathrm{d} \nu
$$

Then the general theory on Green functions allows us to say that it holds $\phi(x)=\int_{\Omega} G(x, y) \nu(\mathrm{d} y)$. Integrating once more with respect to $\nu$ gives the thesis.

Now we will take $\Omega=(-R, R) \subset \mathbb{R}$ and we will divide the square $(-R, R) \times(-R, R)$ into two triangles:

$$
\begin{gathered}
T^{+}=\{(x, y) \in(-R, R) \times(-R, R) \mid x<y\} \\
T^{-}=\{(x, y) \in(-R, R) \times(-R, R) \mid x>y\} .
\end{gathered}
$$

Theorem 8.2. If $\Omega=(-R, R) \subset \mathbb{R}$ and if $V$ is a convex function of the pair $(x, y)$ then the functional $J$ is strictly displacement convex. Consequently, it admits an unique minimizer.

Proof. An easy computation shows that the Green function in (8.1) is given, in the case of the interval $(-R, R)$, by

$$
G(x, y)= \begin{cases}\frac{\cosh (x+R) \cosh (y-R)}{\sinh (2 R)} & \text { if }(x, y) \in T^{+}, \\ \frac{\cosh (x-R) \cosh (y+R)}{\sinh (2 R)} & \text { if }(x, y) \in T^{-}\end{cases}
$$

denoting by cosh and sinh the hyperbolic cosin and sin, respectively. It is also easy to check that both expressions, the one valid in $T^{+}$and the one in $T^{-}$, are strictly convex functions.

Let us now consider a displacement interpolation $\nu_{t}=[(1-t) I d+t T]_{\#} \nu$ and take

$$
J\left(\nu_{t}\right)=\int_{-R}^{R} \int_{-R}^{R}(G+V)(x+t(T(x)-x), y+t(T(y)-y)) \nu(\mathrm{d} x) \nu(\mathrm{d} y) .
$$

Since $T$ must be an optimal transport with respect to the cost $|x-y|^{2}$ it is well-known that it is a nondecreasing map: consequently $(x, y) \mapsto((1-t) x+t T(x),(1-t) y+T(y))$ sends each of the triangles $T^{+}, T^{-}$into itself. Then, in order to get $t \mapsto J\left(\nu_{t}\right)$ strictly convex, it is sufficient to have strict convexity of $G+V$ in each triangle. Our hypothesis ensures it and we get the thesis.

Remark 8.1. In the assumptions of Theorem 8.2 the convexity in each triangle $T^{+}, T^{-}$of $G+V$ is sufficient: in particular, also some concave functions $V$ are allowed.

\subsection{The case of a quadratic kernel in two dimensions}

We now develop the particular case where $V(x, y)=|x-y|^{2}$. For such a choice for $V$ and particular $\Omega$ we are able to give an almost explicit solution. 
First, we make some general considerations on the quadratic kernel. Notice that, for every probability measure $\nu$, we have:

$$
\begin{aligned}
& T_{\nu}(x)=|x-\operatorname{bar} \nu|^{2}+\operatorname{Var} \nu, H(\nu)=2 \operatorname{Var} \nu, \\
& J(\nu)=\|\nu\|_{H^{1}(\Omega)^{\prime}}^{2}+2 \int_{\Omega}|x|^{2} \nu(\mathrm{d} x)-2|\operatorname{bar} \nu|^{2},
\end{aligned}
$$

denoting by bar and Var the barycenter and the variance of a probability measure, respectively.

We also compute the variation of our functional $J$ when we pass from $\nu$ to $\nu+h$, being $h$ an admissible perturbation, i.e. $h=p-\nu$ with $p \in \mathcal{P}(\bar{\Omega})$ :

$$
J(\nu+h)=J(\nu)+2 \int_{\Omega}\left(\phi+T_{\nu}\right) \mathrm{d} h+\|h\|_{H^{1}(\Omega)^{\prime}}^{2}+\int_{\Omega \times \Omega}|x-y|^{2} h(\mathrm{~d} x) h(\mathrm{~d} y) .
$$

By using that $h$ is a zero-mean signed measure, we may re-write the last term and get

$$
J(\nu+h)=J(\nu)+2 \int_{\Omega}\left(\phi+T_{\nu}\right) \mathrm{d} h+\|h\|_{H^{1}(\Omega)^{\prime}}^{2}-\left|\int_{\Omega} x h(\mathrm{~d} x)\right|^{2} .
$$

\subsubsection{The case of the whole space}

In the case $\Omega=\mathbb{R}^{2}$ it is clear that we face a lot of symmetries, with respect both to rotations and to translations. This second kind of symmetries enables us to consider just the problem where the barycenter of $\nu$ is fixed at 0 . Given the set of minimizers for this sub-problem, we will get all the minimizers for the original problem by translating them of an arbitrary vector in $\mathbb{R}^{2}$.

The problem

$$
\inf \left\{J(\nu): \nu \text { probability measure on } \mathbb{R}^{2} \text {, bar } \nu=0\right\}
$$

thanks to $(8.2)$ or $(8.3)$, turns out to be a strictly convex minimization problem. We will then find its unique minimizer by finding a measure $\nu$ satisfying the optimality condition, i.e. such that $x \mapsto \phi(x)+|x|^{2}$ is minimal $\nu$-almost everywhere. Equation (8.3) can be used to convince oneself that such a condition is in fact sufficient to have a minimum.

We will build a solution to the minimization problem by looking for a radial measure with radial potential satisfying proper conditions. The following useful result is given without proof because it is only a (nontrivial, we must admit) second-year exercise.

Lemma 8.3. Consider the Cauchy problem

$$
\left\{\begin{array}{l}
t u_{a}^{\prime \prime}(t)+u_{a}^{\prime}(t)=\frac{u_{a}}{4} \quad \text { for } t \in(a,+\infty) \\
u_{a}(a)=C_{a}-a \\
u_{a}^{\prime}(a)=-1
\end{array}\right.
$$

depending on a parameter $a \in\left(a^{-}, a^{+}\right)$, where $C_{a}$ is a decreasing function of a in the interval $\left(a^{-}, a^{+}\right)$and the following conditions hold: $\lim _{a \rightarrow a^{+}} C_{a}-a<0$ and $\lim _{a \rightarrow a^{-}} C_{a}=+\infty$. Then there exists a number $\bar{a} \in\left(a^{-}, a^{+}\right)$ such that:

- for $a<\bar{a}$ the solution $u_{a}$ is convex and decreasing up to a point $T(a)$ where $u_{a}(T(a))>0$ and $u_{a}^{\prime}(T(a))=$ 0 and then increasing with non-zero derivative;

- for $a=\bar{a}$ the solution $u_{a}$ is convex, decreasing and positive on the whole $(a,+\infty)$ and it is infinitesimal together with its derivative as $t \rightarrow+\infty$;

- for $a>\bar{a}$ the solution becomes negative. 
Moreover, the map $a \mapsto T(a)$ is increasing and continuous and it holds

$$
\lim _{a \rightarrow \bar{a}} T(a)=+\infty
$$

Theorem 8.4. The unique solution to problem (8.4), and so to the whole minimization problem in $\mathbb{R}^{2}$ (up to translations) can be obtained by using lemma 8.3, with $a^{-}=0$, arbitrary large $a^{+}$and

$$
C_{a}=\frac{1+\frac{\pi}{2} a^{2}}{\pi a}-4
$$

Then the solution is the measure $\nu$ whose density is given in the following, together with its potential $\phi$ :

$$
\nu(x)=\left\{\begin{array}{ll}
C_{\bar{a}}+4-|x|^{2} & \text { for }|x|^{2} \leq \bar{a} \\
0 & \text { for }|x|^{2}>\bar{a}
\end{array} ; \phi(x)= \begin{cases}C_{\bar{a}}-|x|^{2} & \text { for }|x|^{2} \leq \bar{a} \\
u_{\bar{a}}\left(|x|^{2}\right) & \text { for }|x|^{2}>\bar{a}\end{cases}\right.
$$

Proof. Thanks to the considerations made before, it is sufficient to check that $\phi$ is the potential of $\nu$ (by computing the laplacian) and that $\nu$ is a probability, i.e. $\int \nu=1$ (but $C$ has been properly chosen); the optimality condition being immediately satisfied by construction $\left(\phi(x)+|x|^{2}\right.$ is constant for $x \in \operatorname{spt} \nu$ and outside it is greater than this constant as a consequence of the convexity of $\left.u_{\bar{a}}\right)$. Similar computations are detailed in the proof of Theorem 8.6.

\subsubsection{The case of a small ball}

The case of a bounded ball in $\mathbb{R}^{2}$ may be interesting as well. In this case, however, we may suffer of a loss of convexity, because we cannot reduce the problem to the simpler one with fixed barycenter. To avoid this difficulty, we will consider a small ball, such that $c_{\Omega, V}<1$. Under this assumption, any critical point of the functional will be actually the unique minimizer. We will build the minimizer exactly as in the case of $\mathbb{R}^{2}$, by using Lemma 8.3. We keep the same choice of $C, a^{-}$and $a^{+}$. By inverting the map $T$ in Lemma 8.3 we define a map $R \rightarrow a(R)$ given by $T(a(R))=R^{2}$ : this map is continuously increasing as well, and it obviously holds $a(R)<R^{2}$.

Theorem 8.5. The unique solution to problem (5.1), when $V(x, y)=|x-y|^{2}, \Omega=B(0, R)$ and $R$ is small enough so that we have $c_{\Omega, V}<1$, is the measure $\nu$ whose density is given in the following, together with its potential $\phi$ :

$$
\begin{aligned}
\nu(x) & = \begin{cases}C_{a(R)}+4-|x|^{2} & \text { for }|x|^{2} \leq a(R) \\
0 & \text { for } a(R)<|x|^{2}<R^{2},\end{cases} \\
\phi(x) & = \begin{cases}C_{a(R)}-|x|^{2} & \text { for }|x|^{2} \leq a(R) \\
u_{a(R)}\left(|x|^{2}\right) & \text { for } a(R)<|x|^{2}<R^{2} .\end{cases}
\end{aligned}
$$

Proof. Just act as in the proof of Theorem 8.4 or have a look at computations in Theorem 8.6.

\subsubsection{The case of a small crown}

Let us now consider a circular crown with radii $R_{1}<R_{2}$, that is the open set $\Omega=B\left(0, R_{2}\right) \backslash \bar{B}\left(0, R_{1}\right)$. To give a solution to the problem we will use once more Lemma 8.3, but this time we will slightly change the function $C$. Moreover, exactly as in the case of the ball, we will only deal with a small crown.

Theorem 8.6. The measure $\nu$ described in the following, together with its potential $\phi$, is the unique solution to problem (5.1), when $V(x, y)=|x-y|^{2}, \Omega=B\left(0, R_{2}\right) \backslash \bar{B}\left(0, R_{1}\right)$ and $R_{1}$ and $R_{2}$ are small enough so that we 
have $c_{\Omega, V}<1$ and $4 \pi R_{1}^{2}<1$ :

$$
\begin{aligned}
\nu & =\nu^{a}+\nu^{s} ; \\
\nu^{a}(x) & = \begin{cases}C_{a\left(R_{2}\right)}+4-|x|^{2} & \text { for } R_{1}^{2}<|x|^{2} \leq a\left(R_{2}\right) \\
0 & \text { for } a\left(R_{2}\right)<|x|^{2}<R_{2}^{2},\end{cases} \\
\nu^{s} & =2 R_{1} \mathcal{H}^{1}\left\llcorner\partial B\left(0, R_{1}\right),\right. \\
\phi(x) & = \begin{cases}C_{a\left(R_{2}\right)}-|x|^{2} & \text { for } R_{1}^{2}<|x|^{2} \leq a\left(R_{2}\right) \\
u_{a\left(R_{2}\right)}\left(|x|^{2}\right) & \text { for } a\left(R_{2}\right)<|x|^{2}<R_{2}^{2},\end{cases}
\end{aligned}
$$

where we have chosen, in Lemma 8.3

$$
C_{a}=\frac{1-4 \pi R_{1}^{2}+\frac{\pi}{2}\left(a^{2}-R_{1}^{4}\right)}{\pi\left(a-R_{1}^{2}\right)}-4
$$

putting $a^{-}=R_{1}^{2}$, and choosing a $\left(R_{2}\right)$ so that it satisfies $T\left(a\left(R_{2}\right)\right)=R_{2}^{2}$.

Proof. This time we give a quite detailed proof. We start by computing the mass of $\nu$ to show that it is a probability on $\bar{\Omega}$.

$$
\begin{aligned}
\nu(\bar{\Omega}) & =\nu^{s}(\bar{\Omega})+\nu^{a}(\bar{\Omega})=4 \pi R_{1}^{2}+\int_{R_{1}}^{\sqrt{a\left(R_{2}\right)}}\left(C_{a\left(R_{2}\right)}+4-\rho^{2}\right) 2 \pi \rho \mathrm{d} \rho \\
& =4 \pi R_{1}^{2}+\left(C_{a\left(R_{2}\right)}+4\right) \pi\left(a\left(R_{1}\right)-R_{1}^{2}\right)-\frac{\pi}{2}\left(a\left(R_{1}\right)^{2}-R_{1}^{4}\right)=1 .
\end{aligned}
$$

To show that $\phi$ is the potential of $\nu$ we divide $\Omega$ into two crowns: $\Omega_{1}=\left\{\left.x \in \Omega\left|R_{1}^{2}<\right| x\right|^{2} \leq a\left(R_{2}\right)\right\}$ and $\Omega_{2}=\left\{\left.x \in \Omega\left|a\left(R_{2}\right)<\right| x\right|^{2}<R_{2}^{2}\right\}$. Then we have, for any $\psi \in C^{1}(\bar{\Omega})$,

$$
\begin{aligned}
\int_{\Omega_{1} \cup \Omega_{2}} \psi \phi+\nabla \psi \cdot \nabla \phi=\int_{\Omega_{1}} \psi(-\Delta \phi+\phi)+\int_{\partial \Omega_{1} \cap \partial \Omega} \psi \frac{\partial \phi}{\partial n}+ & \int_{\partial \Omega_{1} \cap \partial \Omega_{2}} \psi \frac{\partial \phi}{\partial n} \\
& +\int_{\Omega_{2}} \psi(-\Delta \phi+\phi)+\int_{\partial \Omega_{2} \cap \partial \Omega} \psi \frac{\partial \phi}{\partial n}+\int_{\partial \Omega_{2} \cap \partial \Omega_{1}} \psi \frac{\partial \phi}{\partial n} .
\end{aligned}
$$

Let us have a look at the six terms in the right hand side:

- the first one equals $\int_{\bar{\Omega}} \psi \mathrm{d} \nu^{a}$, because in $\Omega_{1}$ we have $\nu^{a}=\phi+4$ and $\Delta \phi=-4$;

- the second term is zero because $\phi$ has, by construction, vanishing normal derivative at $|x|=R_{2}$;

- the third and the sixth one are opposite and so they give a vanishing sum, because $\phi$ is $C^{1}$ by construction;

- the fourth term vanishes because outside $B\left(0, \sqrt{a\left(R_{2}\right)}\right)$ we have $\Delta \phi=\phi$ as a consequence of (8.5);

- the fifth one equals $\int_{\bar{\Omega}} \psi \mathrm{d} \nu^{s}$ by construction of $\nu^{s}$.

After checking that $\phi$ is the potential of $\nu$ it is immediate to notice that, by construction, the optimality conditions are satisfied.

Remark 8.2. Theorem 8.6 gives an example of an optimal $\nu$ composed by an $L^{\infty}$ part and a singular part on $\Gamma_{2}=\partial B\left(0, R_{1}\right)$, while giving no mass to $\partial B\left(0, R_{2}\right)$, which is the convex part of the boundary.

Acknowledgements. The authors have worked on this subject and established all the results during a three months visit period of the second author to GRAPE - Université Montesquieu - Bordeaux IV. The visit has been financially supported 
by the European Research Training Network "Homogenization and Multiple Scales"(HMS2000) under a contract signed with the French CNRS. Grateful acknowledgements go to the Network, the CNRS, and all the people met at GRAPE for their very warm hospitality. A personal acknowledgement goes indeed to Prof. G. Buttazzo for putting the two authors in touch.

\section{REFERENCES}

[1] S. Agmon, A. Douglis and L. Nirenberg, Estimates near the boundary for solutions of elliptic partial differential equations satisfying general boundary conditions I. Comm. Pure Appl. Math. 12 (1959) 623-727.

[2] M. Beckmann, A continuous model of transportation. Econometrica 20 (1952) 643-660.

[3] M. Beckmann and T. Puu, Spatial Economics: Density, Potential and Flow. North-Holland, Amsterdam (1985).

[4] H. Brezis, Analyse Fonctionnelle. Masson Editeur, Paris (1983).

[5] G. Buttazzo and F. Santambrogio, A model for the optimal planning of an urban area. Preprint available at cvgmt.sns.it (2003). To appear in SIAM J. Math. Anal.

[6] G. Buttazzo and E. Stepanov, Optimal transportation networks as free Dirichlet regions for the Monge-Kantorovich problem. Ann. Sc. Norm. Super. Pisa Cl. Sci. 2 (2003) 631-678.

[7] L. De Pascale and A. Pratelli, Regularity properties for Monge transport density and for solutions of some shape optimization problem. Calc. Var. Partial Differ. Equ. 14 (2002) 249-274.

[8] D. Gilbarg and N. Trudinger, Elliptic Partial Differential Equations of Second Order. Springer-Verlag, Berlin (1977).

[9] R.J. McCann, A convexity principle for interacting gases. Adv. Math. 128 (1997) 153-159.

[10] F. Santambrogio, Misure ottime per costi di trasporto e funzionali locali (in italian), Laurea Thesis, Università di Pisa, advisor: G. Buttazzo, available at www. unipi.it/etd and cvgmt.sns.it (2003). 